\title{
The effects of spherical aberration on multiphoton fluorescence excitation microscopy
}

\author{
P.A. Young ${ }^{\star}$, S.G. Clendenon ${ }^{\dagger}$, J.M. Byars ${ }^{\star}$, R.S. Decca ${ }^{\ddagger}$, and K.W. Dunn ${ }^{\star}$ \\ *Department of Medicine, Division of Nephrology, Indiana University School of Medicine, \\ Indianapolis, Indiana, U.S.A \\ †Department of Physics, Indiana University, Bloomington, Indiana, U.S.A \\ ‡Department of Physics, Indiana University-Purdue University Indianapolis, Indianapolis, Indiana, \\ U.S.A
}

\section{Summary}

\begin{abstract}
Multiphoton fluorescence excitation microscopy is almost invariably conducted with samples whose refractive index differ from that of the objective immersion medium, conditions that cause spherical aberration. Due to the quadratic nature of multiphoton fluorescence excitation, spherical aberration is expected to profoundly affect the depth dependence of fluorescence excitation. In order to determine the effect of refractive index mismatch in multiphoton fluorescence excitation microscopy, we measured signal attenuation, photobleaching rates and resolution degradation with depth in homogeneous samples with minimal light scattering and absorption over a range of refractive indices. These studies demonstrate that signal levels and resolution both rapidly decline with depth into refractive index mismatched samples. Analyses of photobleaching rates indicate that the preponderance of signal attenuation with depth results from decreased rates of fluorescence excitation, even in a system with a descannd emission collection pathway. Similar results were obtained in analyses of fluorescence microspheres embedded in rat kidney tissue, demonstrating that spherical aberration is an important limiting factor in multiphoton fluorescence excitation microscopy of biological samples.
\end{abstract}

\section{Keywords}

Multiphoton fluorescence excitation microscopy; refractive index mismatch; spherical aberration; two-photon microscopy

\section{Introduction}

Multiphoton fluorescence excitation microscopy (MPM) has become an invaluable tool enabling biologists to collect fluorescence images up to hundreds of micrometres deep into biological tissues (Helmchen \& Denk, 2005). Although this technique is capable of 
collecting fluorescence images deeper than confocal fluorescence microscopy (Centonze \& White, 1998; Gerritsen \& De Grauw, 1999), the depth to which useful images can be collected is still limited. The limited reach of MPM has been largely attributed to light scattering and absorption (Centonze \& White,1998; Dunn etal., 2000; Oheim et al., 2001; Zipfel et al., 2003; Helmchen \& Denk, 2005; Theer \& Denk, 2006).

However, the reach of MPM for biological imaging has also been shown to be limited by spherical aberration, which significantly affects the quality of MPM fluorescence images (Gerritsen \& De Grauw, 1999; Booth \& Wilson; 2001; de Grauw et al., 2002; Lo et al., 2005; Egner, 2006; Muriello \& Dunn, 2008). Spherical aberration results in the failure of peripheral and axial rays to converge to the same point, thus broadening the size of the focus. By broadening the focus, spherical aberration reduces resolution in wide field microscopy (Hiraoka et al., 1990) and reduces signal in confocal microscopy, due to rejection of emission by the pinhole (Hell et al., 1993). Spherical aberration is also expected to significantly affect MPM because fluorescence excitation is quadratically related to photon density. Therefore the predicted spread in excitation area caused by spherical aberration predicts that this parameter is a critical determinant of loss of fluorescence signal. In fact, the sensitivity of two-photon fluorescence excitation to photon density provides the basis for its inherent optical sectioning capability.

Spherical aberration is a particular problem for microscopy of thick biological samples, in which refractive indices almost invariably differ from that of the immersion medium of the objective lens, a condition that produces increasing amounts of spherical aberration with depth into the tissue (de Grauw et al., 1999). Ironically, the highly localized fluorescence excitation that makes MPM so effective for imaging deep into biological samples would appear to make MPM especially sensitive to the effect of refractive index mismatch characteristic of biological imaging. To some degree the effects of spherical aberration on MPM can be reduced by using an objective lens with a lower numerical aperture (NA), whose smaller angular aperture makes it less sensitive to spherical aberration (Tung et al., 2004). However, the advantage of a low NA objective lens is offset by the increased focus size, which compromises both overall MPM fluorescence excitation and resolution.

By inducing spherical aberration, refractive index mismatch may also compromise resolution of MPM images collected at depth. Although some studies indicate that resolution of MPM decreases with depth into biological tissues (Ying et al., 1999; Schilders \& Gu, 2000), other studies find no such effect (Centonze \& White, 1998; Dunn et al., 2000; Dong et al., 2003).

We expect spherical aberration from refractive index mismatch between the immersion fluid, cover slip and sample to play a significant role in signal attenuation. Here we quantify the effects of refractive index mismatch on signal levels, fluorescence excitation and resolution as a function of depth in both test and biological samples. These studies demonstrate the significance of spherical aberration contribution to signal attenuation at depth in MPM of biological tissues. 


\section{Materials and methods}

\section{Agarose sample preparation}

Samples of agarose were prepared with refractive indices $1.342 \pm 0.002,1.371 \pm 0.001$, $1.404 \pm 0.001,1.442 \pm 0.003\left(22^{\circ} \mathrm{C}\right)$ using varying concentrations of sucrose in NANOpure ${ }^{\circledR}$ water. Suncoast yellow fluorescent microspheres with $02 \mu \mathrm{m}$ diameter (FS02F, Bangs Laboratories, Inc., Fishers, IN, U.S.A.) were suspended in agarose while heated to a liquid state. The agarose then cooled at room temperature to solidify. A vibratome was used to section the agarose to $100 \mu \mathrm{m}$. The refractive index of the sample was measured with an Abbe refractometer (2WAJ, Sino Science \& Technology Co., Ltd, Zhangzhou, Fujian, China).

\section{Tissue preparation}

Animal studies were conducted within the National Institutes of Health Guide for the Care and Use of Laboratory Animals standards. A $250 \mathrm{~g}$ Munich-Wistar rat was anesthetized with pentobarbital (60 $\left.\mathrm{mg} \mathrm{kg}^{-1} \mathrm{IP}\right)$. The femoral vein was then catheterized, and suncoast yellow fluorescent microspheres with $0.2 \mu \mathrm{m}$ diameter (FS02F, Bangs Laboratories, Inc.) were injected. The animal was then killed, and the kidneys were excised. The kidney tissue was fixed by immersion in 4\% paraformaldehyde in phosphate-buffered saline (PBS), pH 7.4, overnight at $4{ }^{\circ} \mathrm{C}$. The tissue was sectioned to $100 \mu \mathrm{m}$ and stored in $0.25 \%$ paraformaldehyde in PBS, pH 7.4, at $4^{\circ} \mathrm{C}$. Before image collection, the tissue was washed overnight in PBS at $4^{\circ} \mathrm{C}$.

\section{Two-photon microscopy}

Samples were imaged using a FV1000 confocal microscope system (Olympus, Center Valley, PA, U.S.A.) that has been adapted for two-photon microscopy. The system is equipped with a Mai-Tai titanium-sapphire laser pumped by a $10 \mathrm{~W}$ Argon laser (SpectraPhysics, Santa Clara, CA, U.S.A.), a Pockels cell beam attenuator (Conoptics Inc., Danbury, CT, U.S.A.) and a Keplerian-style collimator/beam expander fabricated and aligned to fill the back aperture of the objective. The illumination path was aligned using a fluorescent slide to centre the beam and the point-spread functions of suncoast yellow fluorescent microspheres with $0.2 \mu \mathrm{m}$ diameter (FS02F, Bangs Laboratories, Inc.) mounted on a cover slip. We also used a levelling apparatus mounted on the translation stage to ensure the cover slip was perpendicular to the light path (Arimoto, 2004).

Images were collected using either a 60× NA 1.4 oil immersion objective (Olympus) using immersion oil with refractive index 1.515 (Olympus) or a 60× NA 1.2 water immersion objective (Olympus) using water with refractive index 1.33 . The images were $512 \times 512$

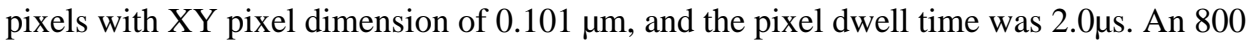
$\mathrm{nm}$ excitation wavelength was used. Fluorescence saturation was avoided, and laser power was maintained constant for all images. Images were collected via descanned detectors with the confocal pinhole open to its maximum. The photomultiplier tube (PMT) gain was constant and below PMT saturation, and PMT black levels were kept constant for all images collected. 


\section{Signal attenuation and resolution degradation image collection}

All samples, agarose and tissue, were mounted with \#1.5 cover slips (Corning, Lowell, MA, U.S.A.). The measured cover slip thickness was $174 \pm 3 \mu \mathrm{m}$. Before mounting, suncoast yellow fluorescent microspheres with $0.2 \mu \mathrm{m}$ diameter (FS02F, Bangs Laboratories, Inc.) were dried to the surface of the cover slip and surface of the slide to delineate the top and bottom of the sample in the image volumes. Seven to 10 image volumes were collected of each sample. The axial step size was $0.10 \mu \mathrm{m}$. Lines were Kalman averaged $\times 2$. The experiment was repeated three times for each sample.

\section{Signal attenuation analysis}

Data were analysed using $\mathrm{IMAGE}_{\mathrm{MAE}}$ (Abramoff et al, 2004) and a custom plugin written at the Indiana Center for Biological Microscopy, which returns the fluorescence intensity of each microsphere, the image plane number of each microsphere and the background intensity of the image volume. Fluorescence intensity of a microsphere was quantified as the average value of the $3 \times 3 \times 3$ voxel region within the image of the microsphere with maximal integrated fluorescence intensity. The image plane number of the microsphere was determined from the centre of this $3 \times 3 \times 3$ voxel region. Background signal in each image volume was determined from the average intensity of the last 10 image planes collected, below the microspheres mounted on the slide at the bottom of the image, and was subtracted from the fluorescence intensity of each microsphere. The depth of each microsphere was measured in micrometres from the cover slip-sample interface, based on the physical movement of the objective, referred to as the nominal focal position (NFP). The NFP is calculated

$$
N F P=z_{\text {step }} *\left(n_{\text {plane }}-n_{\text {offset }}\right)
$$

where $z_{\text {step }}$ is the axial step size $(0.10 \mu \mathrm{m} /$ plane $), n_{\text {plane }}$ is the image plane number of the microsphere and $n_{\text {offset }}$ is calculated by averaging the image plane numbers of all microspheres located at the cover slip. Microspheres were assumed to be located at the cover slip if they were within six image planes of the shallowest microsphere. Axial scaling from refractive index mismatch was not measured.

The data from three trials were combined and averaged in intervals such that there were 25 microspheres per bin. The intervals began at $0.5 \mu \mathrm{m}$ depth to exclude microspheres that were dried on the cover slip and only include microspheres mounted within agarose. Bin widths ranged from 0.61 to $8.00 \mu \mathrm{m}$ wide. Because we attempted to distribute the microspheres homogeneously within the agarose, the bin width was not related to depth in the sample.

The average intensity of the microspheres located at the cover slip was calculated and used to normalize the data. The error for the normalization of the data at the cover slip was calculated 


$$
\left|\delta_{\text {normalization }, n}\right|=\left(\frac{\sqrt{2}}{\langle x\rangle_{\text {coverslip }, n}}\right) * \delta_{\text {coverslip }, n}
$$

Where $\langle x\rangle_{\text {coverslip, } n}$ is the average intensity of the microspheres located at the cover slip for three trials of samples with refractive index $n$ and $\delta_{\text {coverslip, } n}$ is the standard error for the intensity of the microspheres located at the cover slip for three trials of samples with refractive index $n$.

\section{Resolution degradation analysis}

Axial resolution was measured using the image volumes collected of $0.2 \mu \mathrm{m}$ fluorescent microsphere mounted in agarose samples. Although $0.2 \mu \mathrm{m}$ is close to the lateral resolution of the microscope, measured to be $0.3 \mu \mathrm{m}$ using the Olympus $60 \times$ NA 1.4 oil immersion objective, it is much less than the axial resolution, measured to be $0.9 \mu \mathrm{m}$ using $800 \mathrm{~nm}$ twophoton excitation and collected using a $560-660 \mathrm{~nm}$ emission filter, and is therefore a reasonable size for axial resolution analysis.

Data were analysed using $\mathrm{I}_{\mathrm{MAGE}} \mathrm{J}$ (Abramoff et al., 2004) and a custom plugin written at the Indiana Center for Biological Microscopy, which returns pixel values from the line of pixels running axially through the centre of the microsphere and the image plane number of the centre of the microsphere. The centre of the image of the microsphere was determined from the $3 \times 3 \times 3$ voxel region within the image of the microsphere with maximal integrated fluorescence intensity. The NFP was calculated as described in 'Signal attenuation analysis'. The full width at half maximum (FWHM) for the fluorescence intensity distribution from each microsphere was then calculated by curve fitting the data using SIGMAPLot (Systat Software, Inc., Chicago, IL, USA). The data were fit to a four-parameter Gaussian curve

$$
y=y_{0}+a e^{\left[-0.5\left(\frac{x-x_{0}}{b}\right)^{2}\right]} .
$$

The FWHM was then calculated for each Gaussian curve

$$
F W H M=2 b \sqrt{2 \ln (2)} * z_{\text {step }}
$$

The data from three trials were combined and averaged in intervals such that there were 30 microspheres per bin. The intervals began at $0.5 \mu \mathrm{m}$ depth to exclude microspheres that were dried on the cover slip and only include microspheres mounted within agarose. Bin widths ranged from 0.65 to $8.11 \mu \mathrm{m}$ wide.

\section{Excitation attenuation image collection}

Agarose samples were mounted as described above. photobleaching data were collected by first focusing on a microsphere using very low excitation power (as determined by causing undetectable photobleaching after $5 \mathrm{~min}$ of continuous scanning). Once the microsphere was 
in focus, 300 images were collected at approximately 1 frame per second of this single image plane using an Olympus 60× NA 1.4 oil immersion objective. Laser power was measured and maintained constant just below the level that causes fluorescence saturation at the surface of the sample.

The NFP of each microsphere was measured from the cover slip based on the physical movement of the objective. Images were collected of 3-10 microspheres each in $5 \mu \mathrm{m}$ depth intervals in each sample. The experiment was repeated three times for samples with each refractive index.

Fluorescence saturation was assessed by measuring laser power immediately following attenuation of the Pockels cell and then collecting an image of fluorescent microspheres mounted on a \#1.5 cover slip. This was repeated for a range of laser powers to determine at what laser power fluorescence intensity stopped depending quadratically on excitation power, indicating saturation of the fluorophore. Five images were collected at each laser power.

To minimize effects from variable levels of reactive oxygen species from sample to sample, the described agarose samples were mounted in sucrose solutions with $2 \% 1,4-$ Diazabicyclo(2,2,2)octane, a singlet oxygen quencher (Bacallao 2006).

\section{Excitation attenuation analysis}

Photobleaching depends on excitation power and is independent of collection efficiency (Diaspro et al., 2006). Hence, photobleaching was measured as a function of depth to determine the effect of spherical aberration on fluorescence excitation as a function of depth.

For each image plane, the integrated intensity of pixels in a $20 \times 20$ region around a microsphere was recorded using MetaMorph Image Processing software version 4.6r5 (Molecular Devices, Downingtown, PA, U.S.A.). A $20 \times 20$ region was placed in each image that contained no microsphere to measure the integrated intensity of the background. The background was subtracted from the integrated intensity of each $20 \times 20$ region containing a microsphere.

Fluorescence intensity did not decay exponentially during photobleaching, therefore an exponential fit could not be used to determine photobleaching rate. We have confirmed by overlaying curves (data not shown), however, that over long intervals, intensity is a

universal function of $\frac{t}{\tau_{d}}$, where $\mathrm{t}$ is time, and $\frac{1}{\tau_{d}}$ is the depth dependent photobleaching rate. Therefore the intensity at depth $d$ can be written as

$$
I_{d}=I_{0_{d}} f\left(\frac{t}{\tau_{\mathrm{d}}}\right)
$$

where $I_{0}$ is the intensity at $t=0$ seconds. This was done by first averaging the background subtracted intensity versus time data from each microsphere within the field. Intensity versus 
time data were then averaged for each microsphere within a $5 \mu \mathrm{m}$ interval in depth. Intensity versus time data were then normalized to the intensity at $t=0$ seconds. The normalized intensity versus time curve from the image plane collected at the surface of the sample was then scaled in time by multiplying the time axis by an empirical constant $c_{d}$ such as to make the normalized intensity curves for all depths coincide. This constant $c_{d}$ was determined by

$$
c_{d}=\frac{t_{d}}{t_{0}}
$$

where $t_{d}$ is $5 \mathrm{~min}$ of photobleaching at depth $d$ and $t_{0}$ is the time at which

$$
f\left(\frac{t_{0}}{\tau_{0}}\right)=\frac{I_{t_{0}}}{I_{0_{0}}}=\frac{I_{t_{d}}}{I_{O_{d}}}=f\left(\frac{t_{d}}{\tau_{d}}\right)
$$

where $I_{t_{0}}$ is the intensity at the surface of the sample at time $t_{0}, I_{t_{d}}$ is the intensity at depth $d$ after $5 \mathrm{~min}$ of photobleaching. Therefore, $t_{0}$ is the time necessary to photobleach the surface of the sample such that the normalized intensity at the surface $\frac{I_{t_{0}}}{I_{O_{0}}}$ is equal to $\frac{I_{t_{d}}}{I_{O_{d}}}$, the normalized intensity after $5 \mathrm{~min}$ of photobleaching at depth $d$. Therefore we found that for each depth there exists a constant $c_{d}$ such that the curves overlaid each other, and thus there exists a universal curve $f\left(\frac{t}{\tau_{d}}\right)$ valid for all depths. This was repeated for each depth in $5 \mu \mathrm{m}$ intervals.

Because there exists a universal curve valid for all depths, we can determine the depth dependence of the photobleaching rate $\frac{1}{\tau_{d}}$ in our sample. For each depth $d$ the photobleaching rate was normalized to its surface value as

$$
\frac{\tau_{0}}{\tau_{d}}=\frac{t_{0}}{t_{d}}=\frac{1}{c_{d}}
$$

where $t_{d}=5 \mathrm{~min}$. Hence, $\frac{\tau_{o}}{\tau_{d}}$ is equivalent to the ratio of scales in the time axis to make $\frac{I_{t_{d}}}{I_{0_{d}}}$ curves for all depths coincide. Normalized photobleaching rates were averaged from the three trials.

\section{Excitation attenuation calibration data collection}

Calibration data was measured using fluorescent microspheres dried on a \#1.5 cover slip (Corning). The laser power was measured immediately following attenuation by the Pockels cell. Photobleaching data were collected as described above for five different fields at each laser power over a range below fluorescence saturation. The experiment was repeated three times. 


\section{Emission attenuation analysis}

Signal attenuation with depth results from the combined effect of the depth-dependent decrease in excitation of fluorescence and the decrease in collection of fluorescence emissions. By measuring signal attenuation with depth and excitation attenuation with depth, emission attenuation with depth can be estimated from

$$
I_{s}=I_{e}^{2} * I_{c}
$$

where $I_{s}$ is signal intensity normalized to the surface of the sample, $I_{e}$ is the excitation intensity calculated from the photobleaching rate and normalized to the surface of the sample and $I_{C}$ is the corresponding collection intensity normalized to the surface of the sample.

To calculate the excitation intensity $I_{e}$ from the photobleaching rate, the excitation calibration data was plotted on a $\log -\log$ scale, and a linear fit was used to determine the power relationship between the photobleaching rate and the excitation power

$$
\begin{gathered}
R=I_{e}^{p} \\
\log R=p \log I_{e}
\end{gathered}
$$

where $R$ is the photobleaching rate, $I_{e}$ is the excitation intensity and $p$ is the power relationship. Excitation intensity versus depth could then be calculated from the photobleaching rate versus depth measurements. Because excitation is nonlinear based on a two-photon process, the excitation intensity is then squared for direct comparison to the measured signal attenuation with depth.

The fluorescence signal intensity was averaged for 20 microspheres centred at every $5 \mu \mathrm{m}$ depth into the sample and normalized to the average fluorescence intensity at the surface of sample. Bin widths varied from 2.53 to $5.44 \mu \mathrm{m}$ wide.

The fraction of signal attenuation with depth that can be attributed to emission attenuation is thus calculated

$$
I_{c}=\frac{I_{s}}{I_{e}^{2}}
$$

for $5 \mu \mathrm{m}$ intervals into the sample. 


\section{Kidney tissue analysis}

The fluorescence signal intensity was averaged for 20 microspheres centred at every $5 \mu \mathrm{m}$ depth into the sample and normalized to the average fluorescence intensity at the sample surface. Fluorescence intensity at the surface of the sample was calculated as described in 'Signal attenuation analysis'. Bin widths varied from 2.07 to $6.10 \mu \mathrm{m}$ wide for data collected with the oil immersion objective and 1.71 to $11.68 \mu \mathrm{m}$ wide for data collected with the water immersion objective.

\section{Analysis of outliers}

Data were omitted from analysis if fluorescence from the microsphere saturated the detector, or if the fluorescence intensity was greater than twice that of other microspheres at identical depth, indicating the microsphere was an aggregation. The data were tested statistically to eliminate questionable outliers (Rabinovich, 2000).

\section{Results and discussion}

\section{Signal attenuation}

Because two-photon fluorescence excitation is quadratically related to photon density, spherical aberration would be expected to diminish fluorescence excitation at depth into refractive index mismatched samples. In order to isolate the effects of spherical aberration on multiphoton fluorescence excitation, test samples were prepared with minimal light scattering and absorption to assess the effects of refractive index mismatch between the immersion medium, cover slip and sample. Fluorescent microspheres were suspended in samples of agarose, prepared with different refractive indices using sucrose solutions. Images were collected with a $60 \times$ oil immersion objective NA 1.4 . The average intensity of the microspheres located at the cover slip was calculated and used to normalize the data. Data from individual microspheres at the cover slip are not plotted to improve visualization because among the 12 trials there are 597 individual microspheres at the surface of the samples (Fig. 1). The error for the normalization of the data at the cover slip was calculated to be $2 \%$ for each of the four samples.

In all cases fluorescence intensity decreased with depth. However, as expected, fluorescence intensity did not decrease as quickly in samples with refractive index closer to the refractive index of the immersion oil, 1.515. In fact, at $40 \mu \mathrm{m}$ depth into the sample there is a 3.5 -fold improvement in fluorescence signal in a sample with refractive index 1.442 compared to a sample with refractive index 1.342 .

Refractive index mismatch not only broadens the focal point, but it also causes a focal shift (Egner, 2006). In all samples, because the refractive index is less than that of the immersion oil, the focal point will be increasingly offset towards the cover slip with imaging depth into the sample. In samples with refractive index 1.342, this offset is larger than in samples with refractive index 1.442. Therefore, the NFP, the measurement of depth based on the physical movement of the stage, overestimates the actual focal position. By scaling depth using a paraxial approximation, the ratio of the refractive index of the sample to the refractive index of the immersion oil (Wiersma \& Visser, 1996), the effect of refractive index mismatch on 
signal becomes even more pronounced (Fig. 1 - inset). Using the paraxial approximation to scale imaging depth, at $40 \mu \mathrm{m}$ depth into a sample with refractive index 1.442, there is a 3.9fold improvement in fluorescence signal over a sample with refractive index 1.342.

\section{Resolution degradation}

The effects of spherical aberration on resolution are hard to predict knowing that decreased photon flux leads to decreased excitation. Axial resolution was measured as the FWHM of the intensity distributed axially through the centroid of point-spread functions of subresolution microspheres mounted in agarose samples. At the surface of the samples the axial resolution was measured with standard error to be $0.893 \pm 0.005,0.908 \pm 0.006,0.870 \pm 0.014$ and $0.928 \pm 0.005 \mu \mathrm{m}$ (mean \pm standard error) for the samples with refractive index 1.342, 1.371, 1.404 and 1.442, respectively. Data from individual microspheres at the cover slip are not plotted to improve visualization, only the average FWHM (Fig. 2).

In all cases axial resolution degrades with imaging depth. However, the effect of depth on resolution is less pronounced in samples whose refractive index is closer to that of the immersion oil. Axial resolution degrades to $1.7 \mu \mathrm{m}$, nearly 2 fold worse, at $100 \mu \mathrm{m}$ of depth into the sample with refractive index 1.442 but in less than $30 \mu \mathrm{m}$ of depth into the sample with refractive index 1.342. At $100 \mu \mathrm{m}$ depth into the sample with refractive index 1.342, axial resolution is $2.5 \mu \mathrm{m}$, nearly 3 -fold worse than at the surface.

\section{Excitation attenuation}

Because MPM relies on a two-photon process, fluorescence excitation is highly dependent on photon density. Therefore, we expect the preponderance of the signal attenuation with depth in a refractive index mismatched sample to result from decreased fluorescence excitation with depth. Because it is not possible to directly measure the rate of fluorescence excitation, the effect of refractive index mismatch between the immersion medium, cover slip and sample on two-photon excitation was assessed indirectly. This was done by measuring the effect of depth on photobleaching rates, which do not depend on fluorescence collection, only fluorescence excitation. Quantification of the rate of photobleaching of fluorescent microspheres mounted in agarose samples show that photobleaching rates decrease with imaging depth (Fig.3). However, the rate of photobleaching at depth is higher in samples with refractive index closer to that of the immersion oil. At $30 \mu \mathrm{m}$, the sample with refractive index 1.442 has over 7 -fold greater photobleaching than the sample with refractive index 1.342. The increase in photobleaching rate at depth in samples with less refractive index mismatch reflects that refractive index mismatch highly affects fluorescence excitation.

\section{Emission attenuation}

Based on first principles, signal attenuation with depth is caused by attenuation of fluorescence excitation and decreased collection efficiency of fluorescent emissions. Therefore by measuring signal attenuation and excitation attenuation with depth, the contribution of emission attenuation can be inferred. Excitation attenuation is shown to be the predominant contributor to signal attenuation (Fig. 4). At $40 \mu \mathrm{m}$ depth into the sample, only $20 \%$ of fluorescence is excited compared to fluorescence excitation at the surface of the 
sample. Therefore, fluorescence excitation is attenuated by nearly $80 \%$. However, $40 \%$ of fluorescence emissions were collected at $40 \mu \mathrm{m}$ depth into the sample compared to collection at the surface of the sample. Therefore, collection of emissions is attenuated by only about $60 \%$.

\section{Kidney tissue}

Images were collected of kidney tissue mounted in PBS, with refractive index 1.34, using an oil immersion objective. The rate of signal attenuation with depth into kidney tissue was compared to that found in an agarose sample with refractive index 1.342 (Fig. 5). Spherical aberration was shown to cause $88 \%$ of signal to attenuate at $30 \mu \mathrm{m}$ depth in a sample with refractive index 1.342. In fixed kidney tissue mounted in PBS, refractive index 1.34, signal attenuated by $98 \%$ at $30 \mu \mathrm{m}$ depth, indicating $82 \%$ more signal attenuated that was not due to spherical aberration and was likely due to scattering.

Water immersion objectives have been designed with a correction collar that moves optical elements inside the objective to correct for refractive index mismatch caused by the glass cover slip. By collecting images with a water immersion objective, refractive index mismatch between the immersion fluid, cover slip and sample is reduced, decreasing the effect of spherical aberration (Fig. 6). The signal is significantly improved in the first $25 \mu \mathrm{m}$ of depth; however, by $40 \mu \mathrm{m}$ depth the improvement is negligible.

\section{Conclusions}

Refractive index mismatch was shown to result in a rapid attenuation of signal and degradation of resolution with depth, even in samples lacking significant scattering or absorption. This is likely due to spherical aberration induced broadening of the focal volume. In samples that reduce mismatch in refractive index, fluorescence excitation, signal and resolution were improved.

Excitation attenuation was shown to be the major contributor to signal attenuation with depth in MPM. Using an oil immersion objective, fluorescence excitation attenuated by nearly $80 \%$ at $40 \mu \mathrm{m}$ in depth in agarose samples with refractive index 1.342. This is likely due to the quadratic dependence of fluorescence on excitation power when collecting images with MPM. Therefore optimizing fluorescence excitation as a function of depth is very important in MPM.

Collection efficiency was also shown to depend on spherical aberration. Based on the measured signal attenuation and excitation attenuation, it was shown that collected emissions were attenuated by $60 \%$ at $40 \mu \mathrm{m}$. Using non-descanned detectors improves collection of scattered light, reducing the excitation power and detector gain necessary to generate bright images (Centonze \& White, 1998). To assess how signal attenuation with depth compares between non-descanned and descanned detectors, image volumes were collected of agarose samples, refractive index 1.34. The non-descanned detector is a Gallium Arsenide Phosphide PMT (Hamamatsu H7422P-40, Bridge Water, NJ, USA) mounted on the right side port of an Olympus IX-81 microscope, along with a focusing lens (CVI BFPL-25.4-50.0-C-425-675) and 560-650 nm bandpass filter (Chroma HQ605/90M-2P). 
At $50 \mu \mathrm{m}$ in depth, signal attenuated by $92 \%$, using non-descanned detectors and by $95 \%$, using the descanned detectors with the pinhole open fully (data not shown). Therefore although non-descanned detectors in our system collect over $60 \%$ more scattered light at 50 $\mu \mathrm{m}$ depth than descanned detectors, which only amounts to $3 \%$ more signal at $50 \mu \mathrm{m}$ in depth.

In order to assess the contribution of spherical aberration to signal attenuation in kidney tissue, signal attenuation was compared in kidney tissue and homogeneous test samples of agarose with refractive index of 1.342, which matches the refractive index of the tissue mounting medium, PBS of 1.34 . Whereas signal attenuated by $98 \%$ in kidney, it attenuated by $88 \%$ in a sample largely lacking in scattering and absorption, but similar in refractive index, at $30 \mu \mathrm{m}$ depth into the sample. Therefore, signal attenuated by an extra $82 \%$ due to light scattering and absorption at $30 \mu \mathrm{m}$ depth. By collecting images with the water immersion objective, which closely matches the refractive index of kidney tissue mounted in PBS, fluorescent signal was greatly improved for the first $25 \mu \mathrm{m}$ depth into the sample. However, deeper than $25 \mu \mathrm{m}$ signal improved by very little likely due to scattering and absorption. Therefore although by comparing kidney tissue to test samples, it seemed that spherical aberration and scattering contribute equally to signal attenuation, these data indicate scattering is likely the predominant contributor and further study is necessary. However, we have shown that spherical aberration from refractive index mismatch can be a major contributor to depth-dependent signal attenuation in MPM. Consistent with this, we have also shown that signal attenuation can be improved by use of a water immersion objective.

We and others have shown that improvement in reach can be accomplished by manipulating spherical aberration via objective lens adjustment collars (Lo et al., 2005; Muriello \& Dunn, 2008). More elaborate, but still easily implemented solutions for correcting spherical aberration in MPM include adaptive optics manipulations of the illuminations beam, which has been shown to effectively address a variety of tissue-induced aberrations and thus improve the reach of MPM (Albert et al., 2000; Neil et al., 2000; Rueckel et al., 2006).

\section{Acknowledgments}

This work was supported by a George M. O'Brien award from the NIH (P30 DK 079312-01) and conducted at the Indiana Center for Biological Microscopy.

\section{References}

Abramoff MD, Magelhaes PJ, Ram SJ. Image processing with ImageJ. Biophoton Int. 2004; 11(7):3642.

Albert O, Sherman L, Mourou G, Norris TB, Vdovin G. Smart microscope: an adaptive optics learning system for aberration correction in multiphoton confocal microscopy. Opt Lett. 2000; 25(1):52-54. [PubMed: 18059779]

Arimoto R, Murray JM. A common aberration with water-immersion objective lenses. J Microsc. 2004; 216(1):49-51. [PubMed: 15369482]

Bacallao, RL.; Sohrab, S.; Phillips, C. Guiding principles of specimen preservation for confocal fluorescence microscopy. In: Pawley, JB., editor. Handbook of Biological Confocal Microscopy. Springer; New York: 2006. p. 368-380. 
Booth MJ, Wilson T. Refractive-index-mismatch induced aberrations in single-photon and two-photon microscopy and the use of aberration correction. J Biomed Opt. 2001; 6(3):266-272. [PubMed: 11516315]

Centonze VE, White JG. Multiphoton excitation provides optical sections from deeper within scattering specimens than confocal imaging. Biophys J. 1998; 75(4):2015-2024. [PubMed: 9746543]

de Grauw CJ, Vroom JM, van der Voort HTM, Gerritsen HC. Imaging properties in two-photon excitation microscopy and effects of refractive-index mismatch in thick specimens. Appl Opt. 1999; 38(28):5995-6003. [PubMed: 18324119]

de Grauw, CJ.; Frederix, PLTM.; Gerritsen, HC. Aberrations and penetration in in-depth confocal and two-photon-excitation microscopy. In: Diaspro, A., editor. Confocal and Two-Photon Microscopy: Foundations, Applications, and Advances. Wiley-Liss, Inc.; New York: 2002. p. 153-169.

Diaspro, A.; Chirico, G.; Usai, C.; Ramoino, P.; Dobrucki, J. Photobleaching. In: Pawley, JB., editor. Handbook of Biological Confocal Microscopy. Springer; New York: 2006. p. 690-702.

Dong CY, Koenig K, So PTC. Characterizing point spread functions of two-photon fluorescence microscopy in turbid medium. J Biomed Opt. 2003; 8(3):450-459. [PubMed: 12880351]

Dunn AK, Wallace VP, Coleno M, Berns MW, Tromberg BJ. Influence of optical properties on twophoton fluorescence imaging in turbid samples. Appl Opt. 2000; 39(7):1194-1201. [PubMed: 18338003]

Egner, AH.; Hell, SW. Aberrations in confocal and multi-photon fluorescence microscopy induced by refractive index mismatch. In: Pawley, J., editor. Handbook of Biological Microscopy. Plenium Press; New York: 2006.

Gerritsen HC, De Grauw CJ. Imaging of optically thick specimen using two-photon excitation microscopy. Microsc Res Tech. 1999; 47(3):206-209. [PubMed: 10544335]

Hell S, Reiner G, Cremer C, Stelzer EHK. Aberrations in confocal fluorescence miscroscopy induced by mismatches in refractive index. J Microsc. 1993; 169(3):391-405.

Helmchen F, Denk W. Deep tissue two-photon microscopy. Nat Methods. 2005; 2(12):932-940. [PubMed: 16299478]

Hiraoka Y, Sedat JW, Agard DA. Determination of three-dimensional imaging properties of a light microscope system. Partial confocal behavior in epifluorescence microscopy. Biophys J. 1990; 57(2):325-333. [PubMed: 2317554]

Lo W, Sun Y, Lin SJ, Jee SH, Dong CY. Spherical aberration correction in multiphoton fluorescence imaging using objective correction collar. J Biomed Opt. 2005; 10(3):034006-1-034006-5. [PubMed: 16229650]

Muriello PA, Dunn KW. Improving signal levels in intravital multiphoton microscopy using an objective correction collar. Opt Commun. 2008; 281(7):1806-1812. [PubMed: 19343075]

Neil MA, Juskaitis R, Booth MJ, Wilson T, Tanaka T, Kawata S. Adaptive aberration correction in a two-photon microscope. J Microsc. 2000; 200(Pt 2):105-108. [PubMed: 11106950]

Oheim M, Beaurepaire E, Mertz J. Two-photon microscopy in brain tissue: parameters influencing the imaging depth. J Neurosci Methods. 2001; 111(1):29-37. [PubMed: 11574117]

Rabinovich, SG. Measurement Errors and Uncertainties Theory and Practice. Springer; New York: 2000.

Rueckel M, Mack-Bucher JA, Denk W. Adaptive wavefront correction in two-photon microscopy using coherence-gated wavefront sensing. Proc Natl Acad Sci U S A. 2006; 103(46):1713717142. [PubMed: 17088565]

Schilders SP, Gu M. Limiting factors on image quality in imaging through turbid media under singlephoton and two-photon excitation. Microsc Microanal. 2000; 6(2):156-160. [PubMed: 10742403]

Theer P, Denk W. On the fundamental imaging-depth limit in two-photon microscopy. J Opt Soc Am A Opt Image Sci Vis. 2006; 23(12):3139-3149. [PubMed: 17106469]

Tung CK, Sun Y, Lo W, Lin SJ, Jee SH, Dong CY. Effects of objective numerical apertures on achievable imaging depths in multiphoton microscopy. Microsc Res Tech. 2004; 65(6):308-314. [PubMed: 15662621]

Wiersma SH, Visser TD. Defocusing of a converging electromagnetic wave by a plane dielectric interface. J Opt Soc Am A. 1996; 13(2):320-325. 
Ying J, Liu Feng, Alfano RR. Spatial distribution of two-photon-excited fluorescence in scattering media. Appl Opt. 1999; 38(1):224-229. [PubMed: 18305607]

Zipfel WR, Williams RM, Webb WW. Nonlinear magic: multiphoton microscopy in the biosciences. Nat Biotechnol. 2003; 21(11):1369-1377. [PubMed: 14595365] 


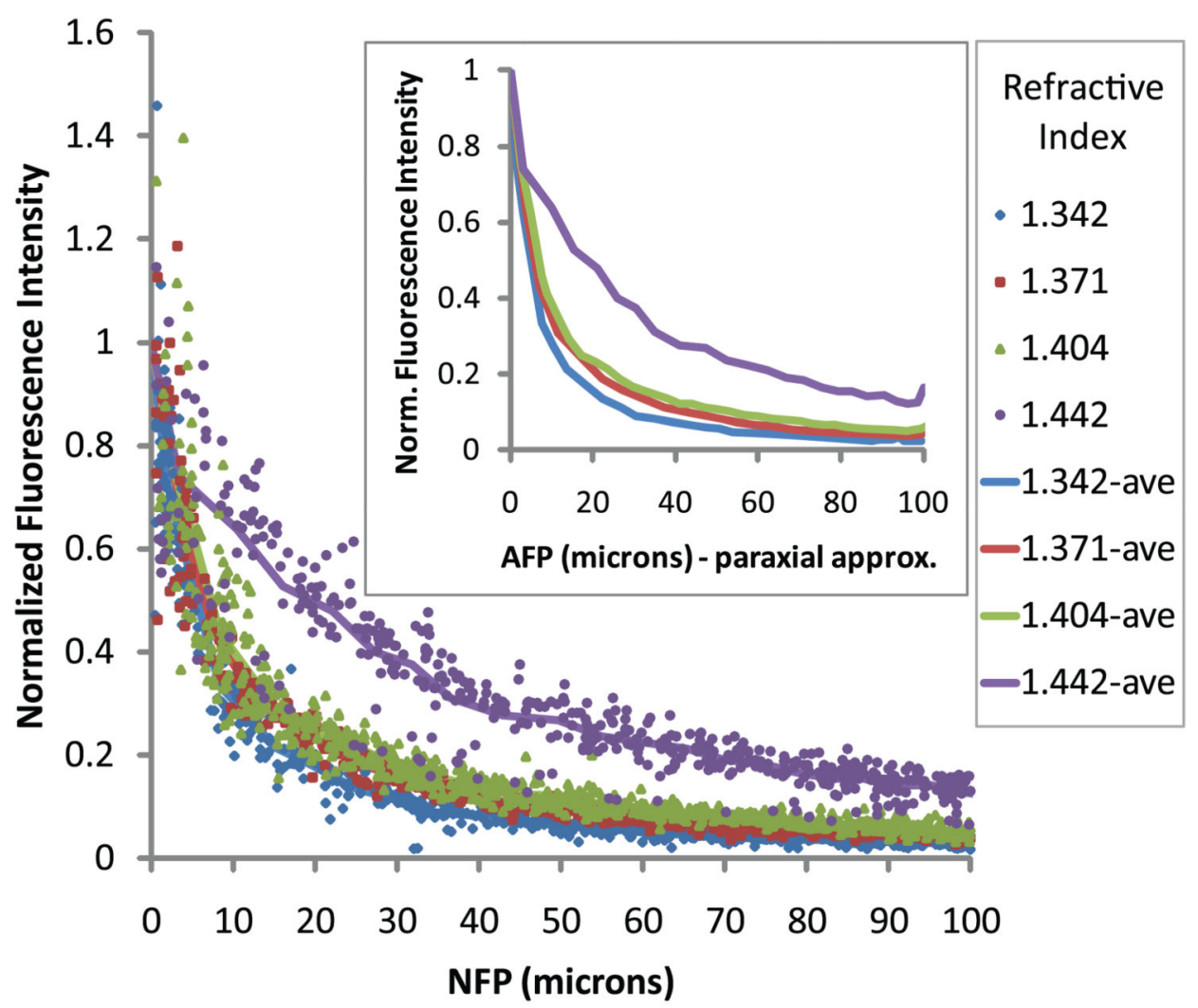

Fig. 1.

Fluorescence intensity of $0.2 \mu \mathrm{m}$ fluorescent microspheres mounted in agarose with varying refractive index, normalized to the average intensity at the surface, versus NFP. Images collected with $60 \times$ oil immersion objective NA 1.4 with immersion oil (refractive index 1.515). Lines represent the average of data from 25 microspheres per bin. Bin widths varied from 0.61 to $8.00 \mu \mathrm{m}$ wide. Inset: Average normalized fluorescence intensity versus depth, where depth has been scaled using the paraxial approximation. 


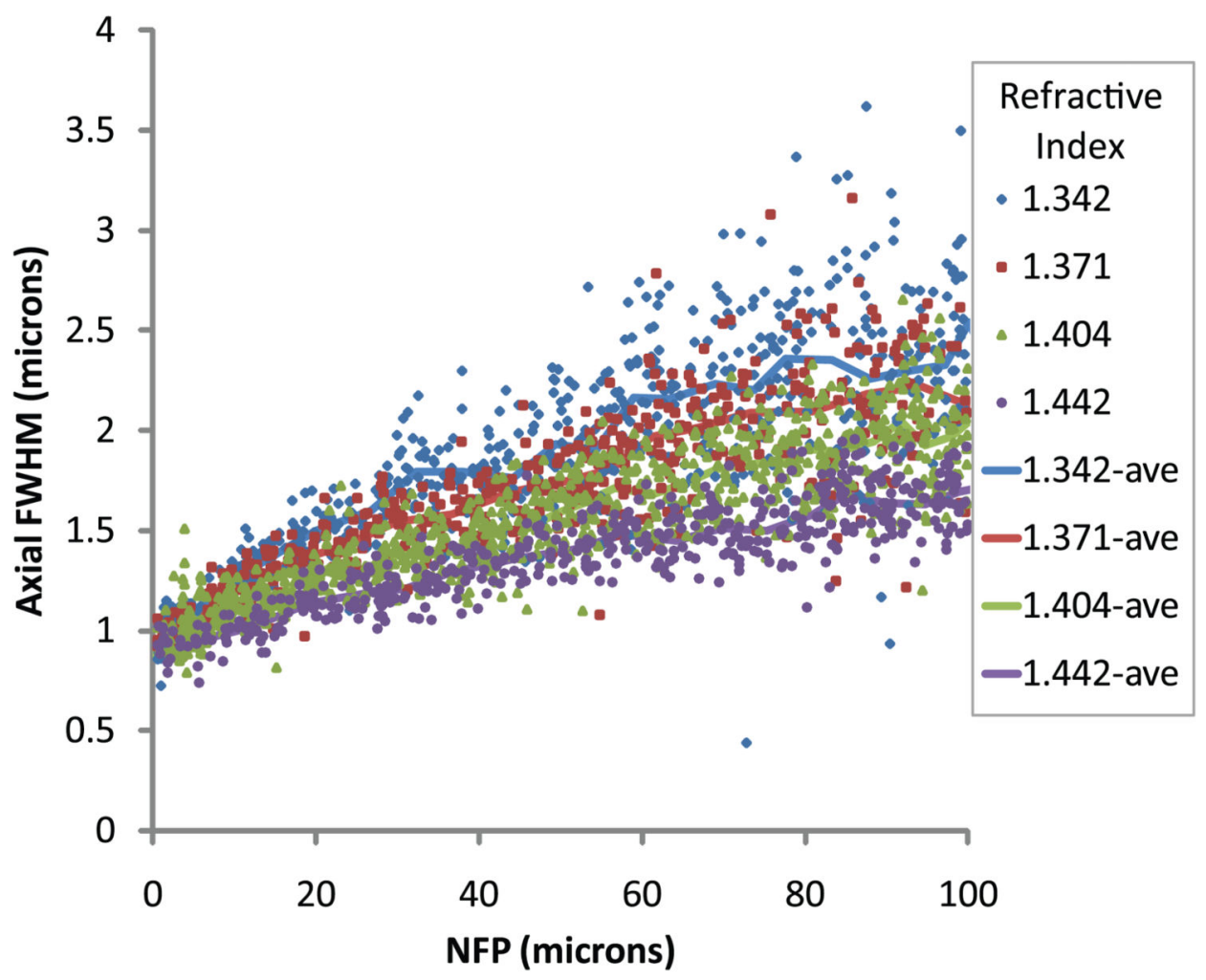

Fig. 2.

Axial intensity distribution FWHM of $0.2 \mu \mathrm{m}$ fluorescent microspheres mounted in agarose with varying refractive index versus NFP. Images collected with $60 \times$ oil immersion objective NA 1.4 with immersion oil (refractive index 1.515). Lines represent the average of data from 30 microspheres per bin. Bin widths varied from 0.65 to $8.11 \mu \mathrm{m}$ wide. 


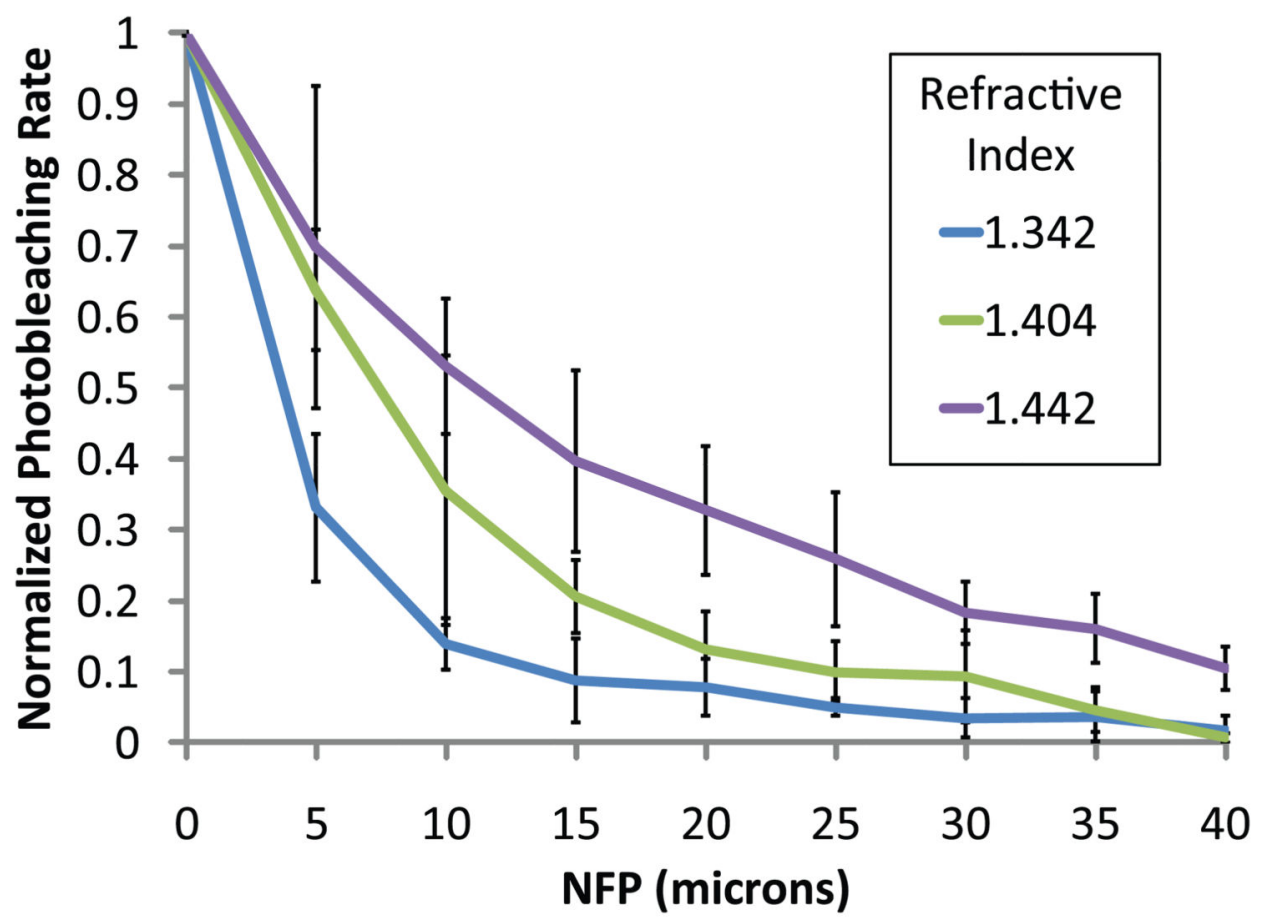

Fig. 3.

Photobleaching rate normalized at the surface of the sample versus NFP. Images were collected with $60 \times$ oil immersion objective NA 1.4 with immersion oil (refractive index 1.515 ) for $5 \mathrm{~min}$ of $0.2 \mu \mathrm{m}$ fluorescent microspheres mounted in agarose samples with varying refractive index. Photobleaching rate was calculated for the average of 10-15 microspheres in $5 \mu \mathrm{m}$ intervals and normalized to the photobleaching rate at the surface. Error bars represent standard deviation of three trials. 


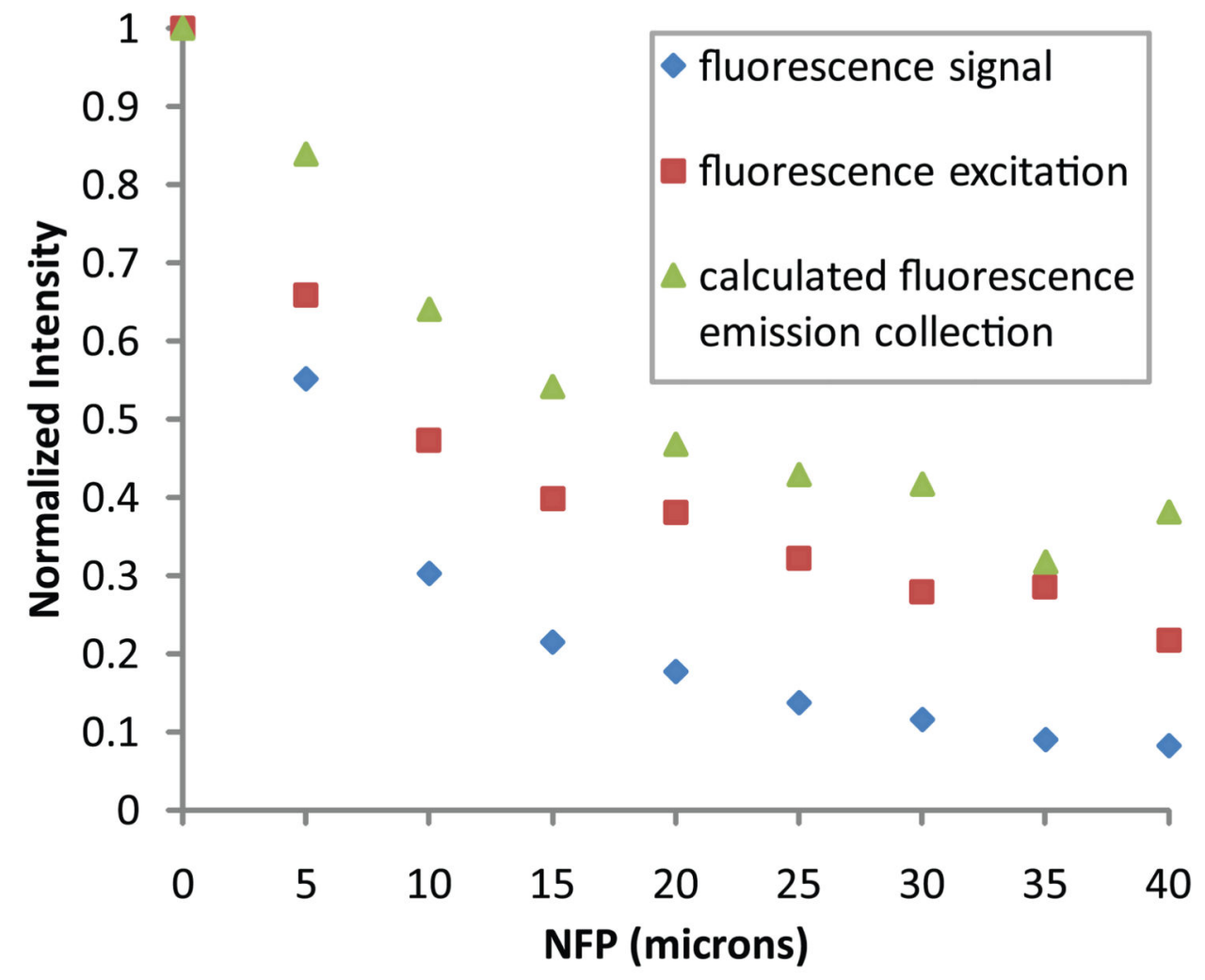

Fig. 4.

Signal attenuation and excitation attenuation versus NFP measured in agarose samples with refractive index 1.342 using a $60 \times$ oil immersion objective NA 1.4 with immersion oil (refractive index 1.515). Signal attenuation data are the average of data from 20 microspheres centred at every $5 \mu \mathrm{m}$ depth and normalized to the average fluorescence intensity at the surface. Excitation attenuation data are calculated from the photobleaching rates at every $5 \mu \mathrm{m}$ depth. Calculated fluorescence emission collection attenuation versus NFP obtained at every $5 \mu \mathrm{m}$ depth. 


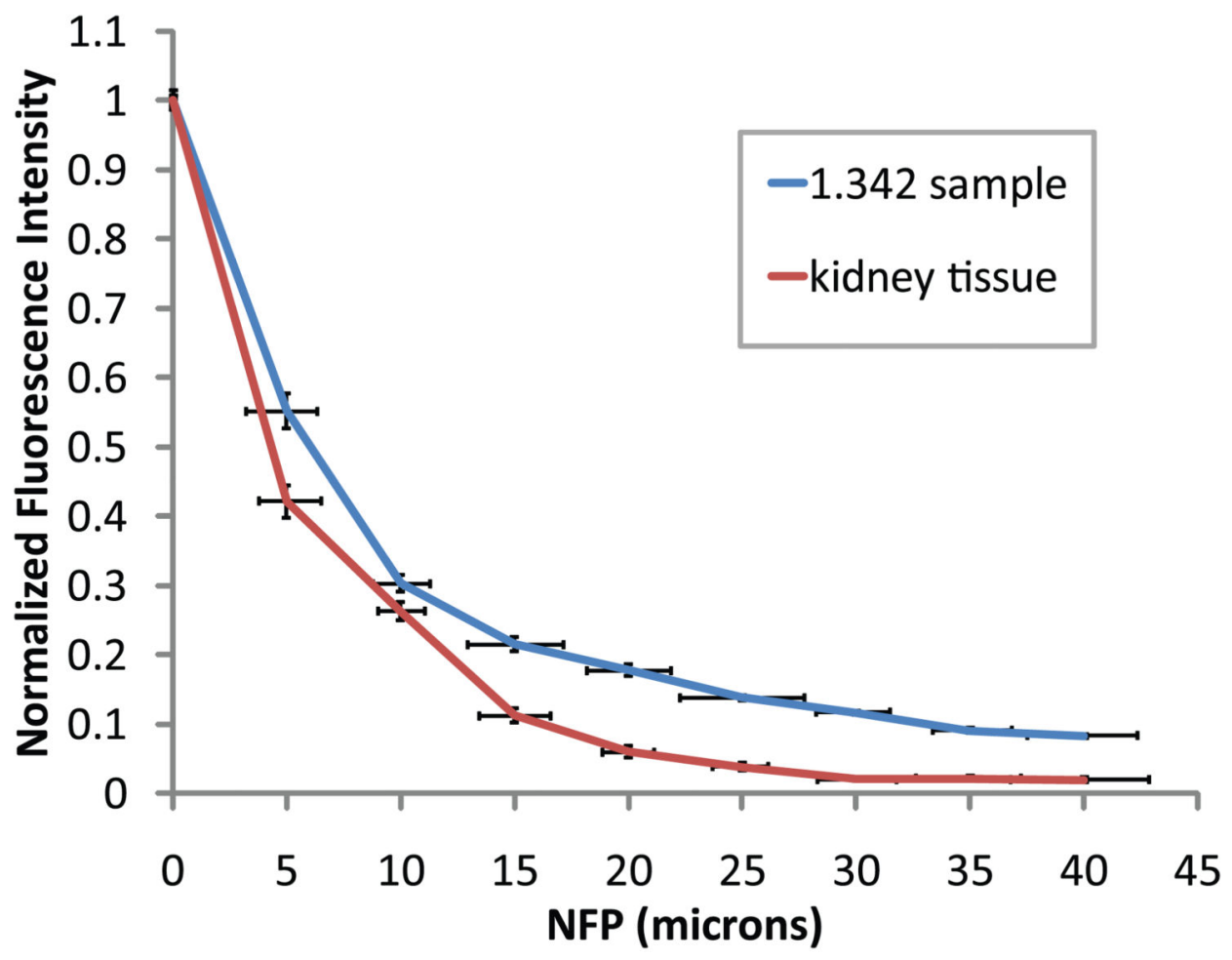

Fig. 5.

Signal attenuation versus NFP of $0.2 \mu \mathrm{m}$ fluorescent microspheres mounted in agarose with refractive index 1.404 or kidney tissue. Images were collected with a $60 \times$ oil immersion objective NA 1.4 with immersion oil (refractive index 1.515).The averages of data from 20 microspheres are centred at every $5 \mu \mathrm{m}$ depth and normalized to the average fluorescence intensity at the surface. Error bars indicate the bin width and standard error. 


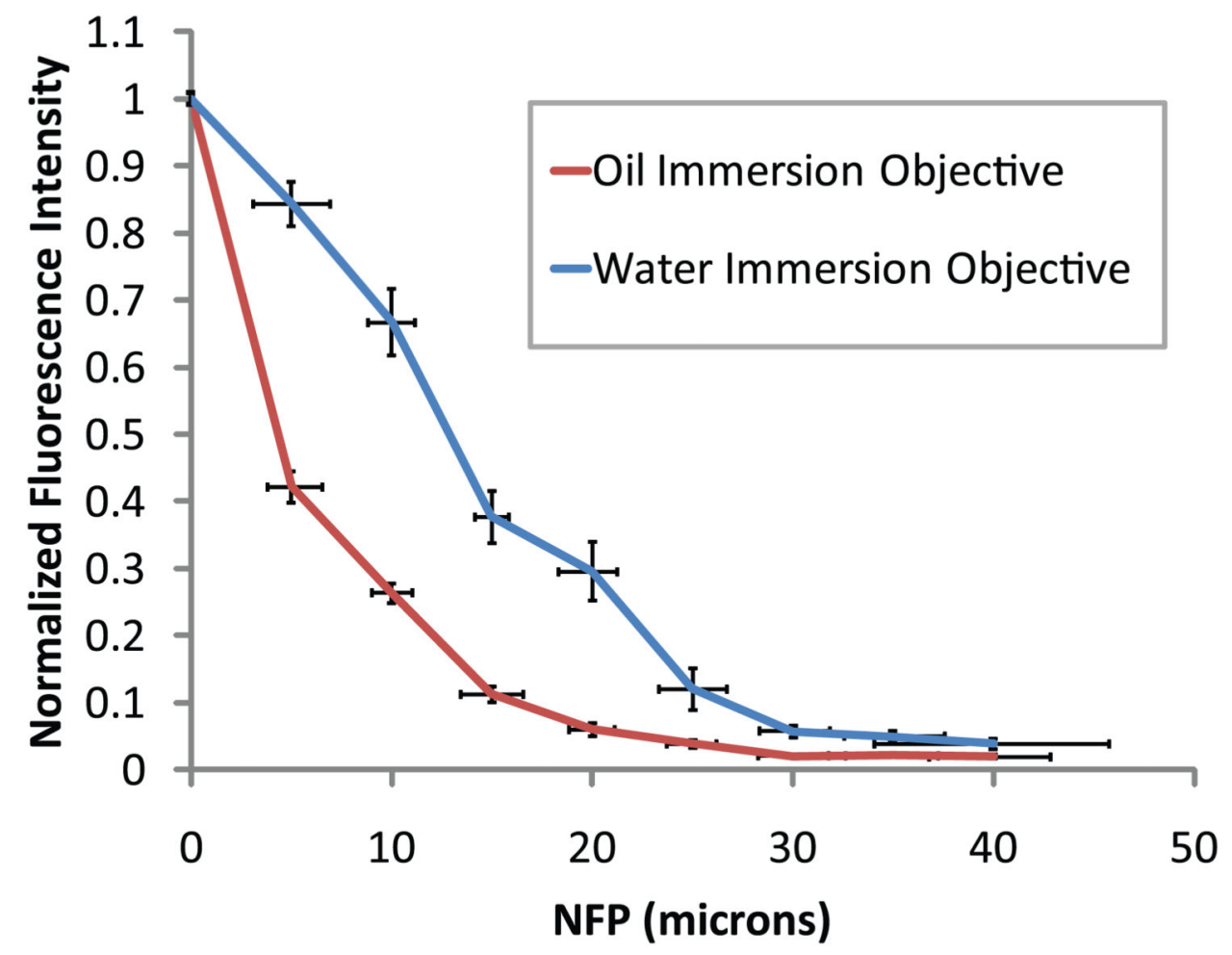

Fig. 6.

Signal attenuation of $0.2 \mu \mathrm{m}$ fluorescent microspheres mounted in kidney tissue. Images were collected with a $60 \times$ oil immersion objective NA 1.4 with immersion oil (refractive index 1.515 ) or a $60 \times$ water immersion objective NA1.2 with immersion water (refractive index 1.33). The averages of data from 20 microspheres are centred at every $5 \mu \mathrm{m}$ depth and normalized to the average fluorescence intensity at the surface. Error bars indicate the bin width and standard error. 